\title{
Role of Coulomb correlations in the optical spectra of semiconductor quantum dots
}

\author{
$\underline{\text { Ulrich Hohenester }}{ }^{(a)}$ and Elisa Molinari \\ Istituto Nazionale per la Fisica della Materia (INFM) and Dipartimento di Fisica \\ Università di Modena e Reggio Emilia, Via Campi 213/A, 41100 Modena, Italy \\ ${ }^{(a)}$ E-mail: hohenester@unimo.it; FAX: +39059367488
}

(May 16, 2000)

\begin{abstract}
We present a consistent theoretical description of few-particle effects in the optical spectra of semiconductor quantum dots, based on a direct-diagonalization approach. We show that, because of the strong Coulomb interaction among electrons and holes, each configuration of the confined fewparticle system leads to its characteristic signature in the optical spectra. We discuss quantitative predictions and comparison with experiments for both absorption and luminescence.
\end{abstract}

PACS: 78.47.+p, 85.30.Vw,71.35.-y,73.20.Dx

KEYWORDS: quantum dot, exciton-exciton interaction

The strong three-dimensional quantum confinement in semiconductor quantum dots (QDs) results in a discrete, atomic-like carrier density of states. In turn, (i) the coupling to the solid-state environment (e.g., phonons) is strongly suppressed [1,2] and (ii) Coulomb correlations among charge carriers are strongly enhanced. Indeed, in the optical spectra of single dots spectrally narrow emission peaks have been observed (indicating a small environment coupling), which undergo discrete energy shifts when more carriers are added to the dot (indicating energy renormalizations due to additional Coulomb interactions) [3].

In this paper we discuss how these spectral changes result from few-particle interactions. A detailed discussion of excitonic and biexcitonic features in the absorption spectra of parabolic QDs is presented; luminescence spectra of multi-excitons and multi-charged excitons are presented, which are compared with experimental data.

The initial ingredients of our calculations are the single-particle states $\phi_{\mu}^{e, h}$ and energies $\epsilon_{\mu}^{e, h}$ for electrons $(e)$ and holes $(h)$, which we obtain by numerically solving the 3D single-particle Schrödinger equation within the envelope-function and effective-mass approximations for arbitrary confinement potentials [4. Next, the fewparticle Hamiltonian (containing all possible $e-e, e-h$, and $h$ - $h$ Coulomb matrix elements) is expanded within the basis of the $\sim 10-20$ energetically-lowest single-particle states, and the few-particle states are obtained by direct diagonalization of the Hamiltonian matrix (see Appendix). For simplicity, in the calculation of the fewparticle $e-h$ states interaction processes with the dot environment are neglected, and only a small broadening of the emission peaks is introduced in the calculation of the optical spectra.

Single excitons. First, we consider the linear optical response. Here, a single electron-hole pair (exciton) is created by an external light field (e.g., laser), which prop- agates in presence of of the dot confinement and of mutual Coulomb interactions. The exciton energies $E_{x}$ and wavefunctions $\Psi_{\mu ; \nu}^{x}$ are obtained from the two-particle Schrödinger equation:

$$
\left(\epsilon_{\mu}^{e}+\epsilon_{\nu}^{h}\right) \Psi_{\mu ; \nu}^{x}+\sum_{\mu^{\prime}, \nu^{\prime}} V_{\mu \mu^{\prime}, \nu \nu^{\prime}}^{e h} \Psi_{\mu^{\prime} ; \nu^{\prime}}^{x}=E_{x} \Psi_{\mu^{\prime} ; \nu^{\prime}}^{x},
$$

with the $e-h$ Coulomb elements $V^{e h}$ defined in Eq. (3) of the Appendix. The optical absorption spectra are then obtained according to Ref. [4] from $\alpha(\omega) \propto$ $\sum_{x}\left|M_{x}\right|^{2} \mathcal{D}_{\gamma}\left(\omega-E_{x}\right)$, where $M_{x}=\sum_{\mu, \nu} \Psi_{\mu ; \nu}^{x} M_{\nu \mu}^{h e}, M^{h e}$ are the optical dipole elements (see Appendix), and $\mathcal{D}_{\gamma}(\omega)=2 \gamma /\left(\omega^{2}+\gamma^{2}\right)$ with a phenomenological damping constant $\gamma$ accounting for interactions with the dot environment.

Figure 1(a) shows the linear optical absorption for a prototypical dot confinement which is parabolic in the $(x, y)$-plane and box-like along $z$ [5]; such confinement potentials have been demonstrated to be a particularly good approximation for various kinds of self-assembled dots [1,6]. We observe a series of pronounced absorption peaks $\left(\bar{X}_{0}, X_{1}, \ldots\right)$ with an energy splitting of the order of the confinement energy; an inspection of the exciton wavefunctions $\Psi_{\mu ; \nu}^{x}$ reveals that the dominant contribution of excitons $X_{0}, X_{1}$, and $X_{2}$ is from the electron and hole single-particle states $1 s, 1 p$, and $(2 s, 1 d)$ (see inset of Fig. 1 $(a))$. In analogy with semiconductor quantum wires 伍, because of Coulomb interactions the energy separation between the groundstate exciton $X_{0}$ and $X_{1}$ is increased, and oscillator strength is transferred from peaks of higher energy to those of lower energy (note that in a pure single-particle picture the ratio of peak heights follows the degeneracy of the respective shells, i.e., $1: 2: 3: 4$ ); finally, for a discussion of the additional Coulomb-induced peaks $\left(X_{0}^{*}, X_{1}^{*}, \ldots\right)$ the reader is referred to Ref. [7,9]. 
Biexcitons. If the dot is populated by two electron-hole pairs, the carrier states available strongly depend on the $e-h$ spin orientations $\left(\sigma_{\uparrow}, \sigma_{\downarrow}\right)$. In the following we only discuss the case of two electrons (holes) with antiparallel spin orientations (for parallel spins see Refs. [8,90). Then, the biexciton energies $\bar{E}_{\lambda}$ and wavefunctions $\Psi^{\lambda}$ are obtained from the $2 e-2 h$ Schrödinger equation (accounting for the various $e-e, e-h$, and $h-h$ Coulomb interactions) which, for conceptual clarity, we write in the exciton ba$\operatorname{sis} x$ 10:

$$
\left(E_{x}+E_{x^{\prime}}\right) \bar{\Psi}_{x x^{\prime}}^{\lambda}+\sum_{\bar{x} \bar{x}^{\prime}} \bar{V}_{x x^{\prime}, \bar{x} \bar{x}^{\prime}} \bar{\Psi}_{\bar{x} \bar{x}^{\prime}}^{\lambda}=\bar{E}_{\lambda} \bar{\Psi}_{x x^{\prime}}^{\lambda}
$$

with $\bar{V}_{x x^{\prime}, \bar{x} \bar{x}^{\prime}}$ the exciton-exciton Coulomb elements [9, 10], and $x, \bar{x}\left(x^{\prime}, \bar{x}^{\prime}\right)$ labeling exciton states with $\sigma_{\uparrow}$ $\left(\sigma_{\downarrow}\right)$. Apparently, the exciton-exciton interaction $\bar{V}$ in Eq. (2) is responsible for the renormalization of the biexciton spectrum. Roughly speaking, the leading contributions to $\bar{V}$ are of dipole-dipole character, with the dipole elements $\mu_{x x^{\prime}}$ according to the excitonic transitions from $x$ to $x^{\prime}\left(\bar{x}\right.$ to $\left.\bar{x}^{\prime}\right)$ [9]; thus, in general both optically allowed and forbidden (due to wavefunction symmetry; see also Fig. 1(b)) excitons with their small and large values of $\mu$, respectively, contribute to $\bar{\Psi}^{\lambda}$.

Figure 2(b) (2(c)) shows optical absorption for a dot which is initially prepared in the $X_{0}\left(X_{1}\right)$ single-exciton state; this scenario of optically probing a non-equilibrium dot is similar to the nonlinear coherent optical response, where a strong pump pulse creates an exciton population at energy $\omega_{p}$ and a weak probe pulse monitors the spectral changes due to the induced exciton population [11]. For the dot initially prepared in state $X_{0}$ (Fig. 2(b)), we observe: At energy $E_{X_{0}}$ negative absorption (i.e., gain) due to the removal of the initial exciton population (i.e., stimulated emission via $\left.X_{0}+h \nu \rightarrow 2 h \nu\right)$; the appearance of an absorption peak $B_{0}$ on the low-energy side of $X_{0}$ and of a peak multiplet (labeled $B_{1}$ ) at spectral position $X_{1}$, attributed to the photo-induced formation of biexcitonic states via $X_{0}+h \nu \rightarrow B$. To a good approximation, the biexciton groundstate $B_{0}$ consists of two groundstate excitons $X_{0}$ with antiparallel spin orientations (because of the small value of $\mu$ for optically allowed excitons the biexciton binding is relatively small); the biexciton states $B_{1}$ consist of $e$ - $h$ pairs in the $1 s$ and $1 p$-shells (see inset of Fig. 1(a)), where the strong mixing with optically forbidden excitons (large $\mu$ 's) leads to large renormalizations and to a strong decrease of the oscillator strengths of the absorption peaks.

Multi-excitons. Next, we turn to the case of a dot populated by a larger number of electron-hole pairs. In a typical single-dot experiment [3], a pump pulse creates $e-h$ pairs in continuum states (e.g., wetting layer) in the vicinity of the QD, and some of the carriers become captured in the QD; from experiment it is known that there is a fast subsequent carrier relaxation due to environment coupling to the $e$ - $h$ states of lowest energy
[2]; finally, electrons and holes in the dot recombine by emitting a photon. By varying in a steady-state experiment the pump intensity and by monitoring luminescence from the dot states, one thus obtains information about the few-particle carrier states. From a theoretical point of view, luminescence involves a process where one $e-h$ pair is removed from the interacting many-particle system and one photon is created. Thus, luminescence spectra provide information about $e-h$ excitations, in contrast to transport measurements of QDs [12] which only provide information about the few-particle groundstate.

Fig. 3(a) shows luminescence spectra for different numbers of $e-h$ pairs (with dot parameters of [5]; luminescence intensity computed according to Ref. [1] and using the few-particle states of Eq. (4) ). We assume that before photon emission the interacting $e-h$ system is in its respective groundstate, i.e., for one $e-h$ pair the exciton groundstate $X_{0}$; for two $e-h$ pairs the state $B_{0}$; for three $e$ - $h$ pairs approximately a filled $1 s$-shell and one $e-h$ pair in the $1 p$-shell, etc.; thus, for one $e$ - $h$ pair luminescence solely originates from the decay of $X_{0}$; for two $e-h$ pairs the biexciton $B_{0}$ decays into $X_{0}$, where the emission peak is slightly red-shifted because of the biexciton binding. In case of three $e-h$ pairs the situation is more involved: For recombination of an $e-h$ pair in the $1 p$-shell, the corresponding luminescence peak is red-shifted by $\approx 10 \mathrm{meV}$ with respect to $X_{1}$ because of exchange corrections of the groundstate energy; for recombination in the $1 s$ shell, after photon emission the dot is in an excited biexciton state; consequently, the peak multiplet in the luminescence spectra is determined by the rather complicated density of states of biexcitonic resonances (see discussion above). Finally, for an increasing number of $e-h$ pairs we observe emission from the $1 s$ and $1 p$ shells, where the peak multiplet from the $1 s$-shell emission exhibits strong spectral changes as a function of the number of $e-h$ pairs. We note that our findings are similar to those obtained in the strong-confinement limit [8] (the difference for the $6 e-6 h$ decay is due to the coupling to higher shells, which are considered in our calculations). Elsewhere [13], it will be shown that our calculated luminescence spectra are in good agreement with experimental single-dot data, with the dots of Ref. [6].

Multi-charged excitons. We finally discuss luminescence spectra of multiple-charged excitons. Experimental realization of such carrier complexes can be found, e.g., in Ref. [14], where GaAs/AlGaAs quantum dots are remotedoped with electrons from donors located in the vicinity of the dot. Employing the mechanism of photo-depletion of the QD together with the slow hopping transport of impurity-bound electrons back to the QD, it is possible to efficiently control the number of surplus electrons in the QD from one to approximately six 14. Fig. 3(b) shows luminescence spectra of charged excitons for a varying number of surplus electrons and for the prototypical dot confinement of [5]. Quite generally, the spectral changes with increasing doping are similar to those presented for multi-exciton states: With increasing doping the main 
peaks red-shift because of exchange and correlation effects. As in the case of multi-exciton states, each fewparticle state leads to a specific fingerprint in the optical response. This unique assignment of peaks or peak multiplets to given few-particle configurations allows to unambiguously determine the detailed few-particle configuration of carriers in QDs in optical experiments; this fact is used in Ref. [14] to study the impurity-dot transport.

Conclusion. We have presented a detailed study of excitonic and biexcitonic features in the optical spectra of a parabolic quantum dot. Luminescence spectra of multi-exciton and multi-charged exciton states have been analyzed, and we have shown that each few-particle configuration leads to its specific fingerprint in the optical response.

Acknowledgements. This work was supported in part by the EU under the TMR Network "Ultrafast" and the IST Project SQID, and by INFM through grant PRASSQI. U.H. acknowledges support by the EC through a TMR Marie Curie Grant.

\section{APPENDIX}

1. Matrix elements. With $\phi^{e, h}$ the single-particle states for electrons and holes, the optical matrix elements

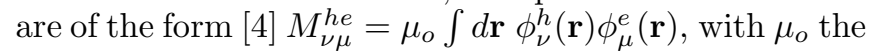
dipole matrix element of the bulk semiconductor. The Coulomb matrix elements read:

$$
V_{\mu^{\prime} \mu, \nu^{\prime} \nu}^{i j}=q_{i} q_{j} \int d \mathbf{r} d \mathbf{r}^{\prime} \frac{\phi_{\mu^{\prime}}^{i}(\mathbf{r}) \phi_{\nu^{\prime}}^{j}\left(\mathbf{r}^{\prime}\right) \phi_{\nu}^{j}\left(\mathbf{r}^{\prime}\right) \phi_{\mu}^{i}(\mathbf{r})}{\kappa_{o}\left|\mathbf{r}-\mathbf{r}^{\prime}\right|}
$$

with $\kappa_{O}$ the static dielectric constant of the semiconductor, $i, j=e, h$ and $q_{e, h}=\mp 1$ (note that we have neglected electron-hole exchange interactions).

2. Few-particle states. We compute the few-particle electron-hole states within a direct-diagonalization approach. With the creation operators $c^{\dagger}$ and $d^{\dagger}$ for electrons and holes, respectively, we define the $N_{e}$-electron and $N_{h}$-hole states $|\vec{\mu}\rangle_{N_{e}}=c_{\mu_{1}}^{\dagger} c_{\mu_{2}}^{\dagger} \ldots c_{\mu_{N_{e}}}^{\dagger}\left|\Phi_{o}\right\rangle$ and $|\vec{\nu}\rangle_{N_{h}}=d_{\nu_{1}}^{\dagger} d_{\nu_{2}}^{\dagger} \ldots d_{\mu_{N_{h}}}^{\dagger}\left|\Phi_{o}\right\rangle$ (vacuum state $\left|\Phi_{o}\right\rangle$; spin degrees of freedom have not been indicated explicitly), and we keep the $\sim 100$ few-particle states of lowest singleparticle energies. Next, the few-particle Hamiltonian $\mathcal{H}$, accounting for all possible electron-electron, electronhole, and hole-hole Coulomb matrix elements, is expanded within these bases; the few-particle energies $E_{\ell}$ and wavefunctions $\Psi_{\vec{\mu} \vec{\nu}}^{\ell}$ are then obtained through direct diagonalization of the Hamiltonian matrix:

$$
E_{\ell} \Psi_{\vec{\mu} ; \vec{\nu}}^{\ell}=\sum_{\vec{\mu}^{\prime}, \vec{\nu}^{\prime}} N_{e} ; N_{h}\left\langle\vec{\mu} ; \vec{\nu}|\mathcal{H}| \vec{\mu}^{\prime} ; \vec{\nu}^{\prime}\right\rangle_{N_{e} ; N_{h}} \Psi_{\vec{\mu}^{\prime} ; \vec{\nu}^{\prime}}^{\ell}
$$

[1] L. Jacak, P. Hawrylak, and A. Wojs, Quantum Dots (Springer, Berlin, 1998).

[2] D. Bimberg, M. Grundmann, and N. Ledentsov, Quantum Dot Heterostructures (John Wiley, New York, 1998).

[3] J. Motohisa, J.J. Baumberg, A.P. Heberle, and J. Allam, Solid-State Electronics 42, 1335 (1998); L. Landin, M.S. Miller, M.E. Pistol, C.E. Pryor, and L. Samuelson, Science 280262 (1998); E. Dekel, D. Gershoni, E. Ehrenfreund, D. Spektor, J.M. Garcia, and M. Petroff, Phys. Rev. Lett. 80, 4991 (1998); F. Vouillez, D.Y. Oberli, F. Lelarge, B. Dwir, and E. Kapon, Solid State Commun. 108, 945 (1998).

[4] F. Rossi and E. Molinari, Phys. Rev. Lett. 76, 3642 (1996); Phys. Rev. B 53, 16462 (1996).

[5] The confinement energies due to the in-plane parabolic potential are $\omega_{o}^{e}=20 \mathrm{meV}$ for electrons and $\omega_{o}^{h}=3.5$ $\mathrm{meV}$ for holes (with this choice, electron and hole wavefunctions have the same lateral extension; we use material parameters for GaAs), and the quantum-well confinement along $z$ is such that the intersubband splittings are much larger than $\omega_{o}^{e, h}$.

[6] R. Rinaldi, P. V. Giugno, R. Cingolani, H. Lipsanen, M. Sopanen, J. Tulkki, and J. Ahopelto, Phys. Rev. Lett. 77, 342 (1996); Phys. Rev. B 57, 9763 (1998).

[7] U. Hohenester, R. Di Felice, E. Molinari, and F. Rossi, Appl. Phys. Lett. 75, 3449 (1999).

[8] P. Hawrylak, Phys. Rev. B 60, 5597 (1999).

[9] U. Hohenester and E. Molinari, phys. stat. sol. (a) 178, 277 (2000).

[10] V.M. Axt and S. Mukamel, Rev. Mod. Phys. 70, 145 (1998).

[11] N.H. Bonadeo, Gang Chen, D. Gammon, D.S. Katzer, D. Park, and D.G. Steel, Phys. Rev. Lett. 81, 2759 (1998).

[12] R. C. Ashoori, Nature 379, 413 (1996); L. P. Kouwenhoven et. al., in: L. Sohn et al. (Eds.), Mesoscopic Electron Transport (Kluwer, Dordrecht, 1997).

[13] R. Rinaldi, S. Antonaci, M. DeVittorio, R. Cingolani, U. Hohenester, E. Molinari, H. Lipsanen and J. Tulkki, Phys. Rev. B (2000), in press.

[14] A. Hartmann, Y. Ducommun, E. Kapon, U. Hohenester, and E. Molinari, Phys. Rev. Lett. (2000), in press; condmat/0005189.

[15] H. Haug, S.W. Koch, Quantum Theory of the Optical and Electronic Properties of Semiconductors (World Scientific, Singapore, 1993). 
FIG. 1. (a) Linear absorption spectrum for dot [5] (computed for a basis of 20 electron and 40 hole single-particle states); the inset shows the single-particle wavefunctions of lowest energy (states $1 p$ are double degenerate, states $1 d$ and $2 s$ are triple degenerate). (b) Contour plot of the exciton wavefunctions $\sum_{x}\left|\Psi^{x}(\mathbf{r}, \mathbf{r})\right|_{\mathbf{r}=(X, 0,0)}^{2} \mathcal{D}_{\gamma}\left(\omega-E_{x}\right)$; because of symmetry only a small portion of the excitons couples to the light [9].

FIG. 2. Optical absorption spectra for QD initially prepared in the (a) vacuum state (i.e., linear absorption), (b) exciton grondstate $X_{0},(c)$ state $X_{1}$ (i.e., nonlinear absorption). For a discussion see text.

FIG. 3. Luminescence spectra for QD and for different (a) multi-exciton and (b) multi-charged excitons states. We assume that before photon emission the electron-hole system is in its groundtstate (i.e., $(a)$ : $\left(1 e_{\uparrow} ; 1 h_{\downarrow}\right),\left(1 e_{\uparrow}, 1 e_{\downarrow} ; 1 h_{\downarrow}, 1 h_{\uparrow}\right)$, $\left(2 e_{\uparrow}, 1 e_{\downarrow} ; 2 h_{\downarrow}, 1 h_{\uparrow}\right), \quad\left(3 e_{\uparrow}, 1 e_{\downarrow} ; 3 h_{\downarrow}, 1 h_{\uparrow}\right), \quad\left(3 e_{\uparrow}, 2 e_{\downarrow} ; 3 h_{\downarrow}, 2 h_{\uparrow}\right)$, and $\left(3 e_{\uparrow}, 3 e_{\downarrow} ; 3 h_{\downarrow}, 3 h_{\uparrow}\right) ; \quad(b): \quad\left(1 e_{\uparrow} ; 1 h_{\uparrow, \downarrow}\right), \quad\left(1 e_{\uparrow}, 1 e_{\downarrow} ; 1 h_{\uparrow, \downarrow}\right)$, $\left(2 e_{\uparrow}, 1 e_{\downarrow} ; 1 h_{\uparrow, \downarrow}\right), \quad\left(3 e_{\uparrow}, 1 e_{\downarrow} ; 1 h_{\uparrow, \downarrow}\right), \quad\left(3 e_{\uparrow}, 2 e_{\downarrow} ; 1 h_{\uparrow, \downarrow}\right)$, $\left.\left(3 e_{\uparrow}, 3 e_{\downarrow} ; 1 h_{\uparrow, \downarrow}\right)\right)$ 
This figure "figure1.jpg" is available in "jpg" format from: http://arxiv.org/ps/cond-mat/0005253v1 
This figure "figure2.jpg" is available in "jpg" format from: http://arxiv.org/ps/cond-mat/0005253v1 
This figure "figure3.jpg" is available in "jpg" format from: http://arxiv.org/ps/cond-mat/0005253v1 